\title{
Enseignement de l'éthique : une approche socratique
}

\author{
Teaching Ethics: A Socratic Approach
}

Résumé Contexte : La Faculté de médecine et des sciences de la santé de l'Université de Sherbrooke implante depuis 2005 une nouvelle stratégie pour l'enseignement de l'éthique clinique qui se différencie d'une formation éthique tributaire du "modèle de rôle ", du "principisme " et du "pragmatisme ". La stratégie proposée est l'approche socratique inspirée par le Socrate de Platon qu'a repris Nelson et son disciple Heckmann dans un cadre pédagogique selon une perspective néokantienne. Exégèse : L'article propose de donner une extension herméneutique à cette approche en mettant en valeur le rôle du dialogue dans la résolution d'un problème éthique, en sáppuyant sur l'expérience humaine dans ses besoins et ses désirs. Dans cette perspective herméneutique, l'enseignement de l'éthique ne consiste pas à inculquer une connaissance des différentes théories éthiques, mais consiste pour l'étudiant à développer sa capacité de discernement au moyen d'une plus grande prise de conscience. Conclusion: Cette approche n'est pas sans conséquence sur le rôle traditionnel dévolu à l'enseignant et à l'étudiant, car l'approche socratique se veut un exercice de subjectivation où l'individu apprend à penser par lui-même à travers la force de décentrement quiexerce la diversité des points de vue présentés par les autres.

Mots clés Ethique clinique ; enseignement ; herméneutique ; dialogue ; approche socratique ; autonomie.

Abstract Background: The Medical and Health Sciences Departments of Sherbrooke University implant since 2005 a new teaching strategy of the clinical ethics which differs from the training dependent on the "role model", the "principlism", as well as pragmatism. The proposed strategy is inspired after the Socrates of Plato, which was retrieved by Nelson and his proselyte Heckmann in a pedagogical field according to the neokantian perspective. Exegesis: This article suggests giving a hermeneutic extension to this approach by emphasizing the role of dialogue for the resolution of an ethical problem while relying on human experience in its needs and its desires. In this hermeneutical outlook, teaching the ethics will not only consist in inculcating the knowledge of the various ethical theories, but will consist of, for the student, to increase its capacity to discern by means of a widened awareness. Conclusion: This approach is not without consequences upon the traditional role devolved to the teacher and the student, for the Socratic approach is an exercise of subjectivity upon which an individual learns to think by himself through an offset force issued from the diversity of points of view presented by others.

Key words Clinical Ethics; teaching; hermeneutics; dialogue; Socratic approach; autonomy.

Pédagogie Médicale 2008;9:166-70

Correspondance: Jacques Quintin, Bureau de développement de l'éthique,Département de psychiatrie -Faculté de médecine et des sciences de la santé, Université de Sherbrooke - Adresse postale : 580 rue Bowen Sud - J1G 2E8 Sherbrooke - Québec (Canada) Téléphone : 819-820-6868 poste 23546. Télécopie : 819-822-6790 - Mailto:jacques.quintin@USherbrooke.ca 


\section{Introduction}

Depuis une vingtaine d'années, notre société vit une crise du savoir. Certes, ce n'est pas la première fois que l'Occident vit une telle crise, mais c'est toutefois la première de ce genre si l'on considère la spécificité de l'époque qui est marquée par un contexte de pluralisme culturel, d'individualisme et de relativisme. Cette crise n'est pas sans incidence sur la définition traditionnelle des enseignants comme transmetteurs de connaissance. C'est la raison pour laquelle des penseurs humanistes comme Michel Serres, Edgar Morin ou l'Américaine Amy Gutmann, "pensent qu'il n'est plus possible de décrire les tâches des enseignants en fonction des contenus et des connaissances $»^{1}$.

Cette transformation est perceptible partout et particulièrement dans l'enseignement de l'éthique, qui est devenu une préoccupation réelle pour les universités et particulièrement les facultés de médecine qui ont fait de l'éthique clinique une priorité. C'est le cas notamment de la Faculté de médecine et des sciences de la santé de l'Université de Sherbrooke, qui implante depuis 2005 une nouvelle stratégie pour l'enseignement de l'éthique clinique. Bien qu'il soit généralement accepté que l'éthique médicale est davantage qu'une simple application extérieure des valeurs morales acceptées socialement, il n'en demeure pas moins que la question de savoir quelles sont les stratégies didactiques les plus appropriées pour l'enseignement de l'éthique demeurent un sujet de controverse.

\section{Problématique}

L'un des problèmes que pose l'enseignement de l'éthique tient à la quantité d'informations à transmettre, la définition de leur type et de leur nature. L'apprentissage de l'éthique passe souvent par l'enseignement des concepts fondamentaux de la philosophie, qui sont habituellement présentés à l'aide des penseurs classiques et contemporains. On oublie trop souvent, cependant, que les étudiants qui ont choisi les sciences, en l'occurrence la médecine, le font, en partie, pour éviter ces matières trop spéculatives, car ils se destinent avant tout à être des praticiens et non des théoriciens.

D’aucuns, en s'inspirant de MacIntyre ${ }^{2}$, défendront l'idée que la pratique médicale s'autorégule car elle possède ses propres valeurs internes telles que la fidélité à la finalité de la médecine, par exemple l'éradication de la maladie ou l'allègement des symptômes, qui sont transmises par l'apprentissage clinique. Dans un article publié au début des années 90, Hafferty et Franks révèlent et discutent le profil presque invisible de l'éthique médicale qui fait de l'éthique un "curriculum caché »".

Parallèlement à la reconnaissance de cette formation éthique tributaire du " modèle de rôle ", d'autres préferent se servir du courant qui repose sur le "principlism " élaboré par Beauchamp et Childress ${ }^{4}$, qui s'est particulièrement imposé depuis les années 80, et qui domine encore largement au sein du milieu de la santé. Pour plusieurs, les quatre principes que sont l'autonomie, la bienfaisance, la non-malfaisance et la justice constituent les principales valeurs impliquées dans la pratique des soins de santé. La critique principale formulée à l'égard de cette approche souligne toutefois, qu'en dépit des recommandations de Beauchamp et de Childress de prendre en considération le contexte particulier de chaque situation, ces principes sont pris trop souvent comme une fin en soi au lieu d'être utilisés comme des outils pour interpréter les situations toujours singulières.

Enfin, le pragmatisme, inspiré par Dewey, qui met l'accent sur une attitude pratique dans la mesure où l'intérêt est davantage porté sur la résolution de problème que sur des discussions théoriques, exerce également une grande influence sur les approches de l'enseignement de l'éthique.

La stratégie qui est proposée a l'avantage de mettre en valeur chacune des stratégies ci-dessus nommées, sans pour autant se réduire à aucune. Si l'éthique clinique cherche en outre à satisfaire les besoins de chacun (professionnel de la santé et patient), il faut alors se doter d'une stratégie éducative qui puisse reproduire la conception selon laquelle il s'agit de commencer par reconnaître autrui dans ses propres besoins et ses désirs. Cette stratégie est l'approche socratique.

\section{L'approche socratique}

Lorsque nous parlons d'une approche socratique, nous ne désignons pas le Socrate tel que nous le retrouvons dans les dialogues de Platon, mais plutôt ce que ce même Socrate inspire : l'idée que les connaissances philosophiques sont essentiellement structurées dans l'expérience humaine. C'est ainsi que ces connaissances sont accessibles à chacun, pour autant que soit fourni un minimum d'effort intellectuel jumelé à de la bonne volonté, pour accoucher des connaissances le plus souvent dissimulées derrière le voile de l'habitude, des illusions, des préjugés et des conventions, bref, de ce qui va de soi et que, par conséquent, l'être humain oublie. Ces 


\section{Concepts et Innovations}

connaissances sont le résultat d'une compréhension qui prend sa source dans les propres ressources de l'étudiant révélées à l'aide de l'enseignant.

Lidée principale de cette approche consiste à encourager les étudiants à réfléchir sur un problème éthique à partir d'eux-mêmes, sans l'aide de livre ou de cadre conceptuel. Il s'agit de fournir un effort intellectuel qui ne s'appuie ni sur des textes fondateurs, ni sur des théories, mais sur l'expérience humaine. Comme Socrate l'a montré dans le Ménon, les connaissances sont structurées dans l'expérience humaine. L'apprentissage, mené comme une enquête, est une réminiscence qui émerge à partir d'une interrogation dialectique où se met en œuvre le jeu de questions et de réponses. Par conséquent, l'apprentissage n'est pas le transfert d'un savoir d'un enseignant à un élève, mais l'expérience d'une prise de conscience. Dès lors, l'effort intellectuel consiste à se remémorer ces connaissances enfouies dans nos expériences.

Contrairement aux dialogues retrouvés chez le Socrate de Platon, dialogues dyadiques entre un maître et un élève, le dialogue adopté dans la méthode socratique est pluriel. Chacun des participants du groupe de discussion y établit un rapport maïeutique, rapport qui assure une fonction de sage-femme, pour les découvertes philosophiques de tous les autres participants. En passant d'un paradigme dyadique à un paradigme de groupe, le rôle de l'enseignant est modifié. Son rôle devient celui de sage-femme au même titre que les autres membres du groupe. Mais son rôle implique aussi un travail de discipline dans la mesure où il s'agit de maintenir la discussion de groupe à l'intérieur des limites de la question posée. Autrement dit, sa tâche consiste à renforcer les règles procédurales permettant un processus de pensée diversifiée, ouverte et orientée vers le consensus.

Les premiers penseurs à avoir proposé une extension des dialogues socratiques à l'enseignement sont le philosophe néokantien Leonard Nelson (1882-1927) ${ }^{5}$ et son disciple Gustav Heckmann (1898-1996) ${ }^{6}$. Selon Nelson, les vérités philosophiques sont à trouver à l'intérieur de nos présupposés quotidiens. Pour ce faire, il propose une méthode de régression qui consiste à chercher les présupposés généraux sous-jacents à notre manière de concevoir le monde et nous-mêmes, qui devraient permettre de découvrir un ensemble de vérités transcendantales. Contrairement à cette approche néokantienne qui donne une validité universelle et absolue à ces vérités, nous proposons une approche de type herméneutique, car nous croyons que ces vérités sont le fruit d'un travail d'interprétation et donc toujours sujettes à une révision selon le contexte et notre histoire.

Cette compréhension herméneutique de la méthode socratique met l'accent sur l'idée que la philosophie, plus spécifiquement l'éthique, est une pratique plutôt qu'une théorie, davantage une activité qu'un corpus de connaissance. Dans cette perspective, l'enseignement de l'éthique ne consiste pas à inculquer une connaissance des différentes théories éthiques ou tout autre contenu spécifique à l'éthique, mais consiste pour l'étudiant à exercer sa capacité de parvenir à un jugement éthique, c'est-à-dire à partir de son propre effort, à identifier un problème, délibérer et parvenir à poser un jugement et, si possible, à un consensus. L'enseignement de l'éthique vise davantage le développement d'habiletés et de compétences que l'acquisition d'informations et de connaissances, ce qui fait que la philosophie, ou l'éthique, est un art et non une science. Nelson fait la remarque qu'il ne s'agit pas d'enseigner la philosophie, mais d'enseigner à philosopher. Loin de l'image caricaturale du philosophe qui observe depuis une tour d'ivoire et pense après les événements et après les autres donc, après coup, le clinicien qui réfléchit, lui, se situe au contraire dans l'action et l'espace public, en tentant de prévoir les coups pour favoriser le meilleur et éviter le pire. À cet égard, le clinicien ou l'homme de terrain fait sienne la maxime de Marx : «Les philosophes n'ont fait qu'interpréter diversement le monde, ce qui importe, c'est de le transformer ". Le vrai penseur, comme le souligne Bergson, est celui qui fait émerger, pour chaque cas singulier, le concept qui lui serait approprié. Gadamer dira que le travail de la pensée consiste à amener au concept ce qui est implicite dans nos actions et nos paroles. Par conséquent, ce n'est pas un objet qui est enseigné, mais un processus, un acte qui trouve sa source à l'intérieur de la personne.

L'exercice de réflexion proposé par l'approche socratique-herméneutique commence donc par des problèmes que les étudiants rencontrent dans leur vie concrète, au lieu de débuter avec des histoires de cas retrouvés dans les textes de base. Il s'agit d'aller chercher les personnes là où elles se trouvent, en les faisant parler pour réactualiser leurs connaissances et les élever à un niveau supérieur de réflexivité par les sentiers du dialogue. Il ne s'agit pas de ressasser de grands principes, de grands concepts, mais de partir des besoins et des désirs de chacun, là où chacun se trouve déjà : si la personne est dans le déni, alors partir du déni. Commencer ainsi par identifier les malaises, les inconforts, les sentiments 
d'indignité pour chacune des personnes impliquées, pour ensuite cibler les besoins de tous et imaginer par la suite des possibilités qui permettraient d'arriver à une solution raisonnable pour tous.

\section{Les règles du jeu}

Pour y parvenir, l'enseignant doit respecter certaines règles. La première de ces règles est celle qui permet l'ouverture concernant les questions soulevées. Ce faisant, la tâche de l'enseignant n'est pas d'enseigner, mais de guider les étudiants vers leur propre conclusion. Si l'enseignant est directif, c'est seulement pour renforcer les règles du jeu puisque son rôle est celui de facilitateur. Son rôle est de créer une atmosphère détendue, chaleureuse et tolérante, motivant ainsi les participants à mener un débat ouvert.

Il y a aussi la règle du respect des gens qui pensent autrement, plus lentement ou qui s'expriment plus difficilement, et qui donne la chance à tous de contribuer. Etant donné que personne ne possède les mêmes capacités, les mêmes habiletés et les mêmes motivations, il revient à l'enseignant de veiller au respect de la parole de tous.

L'enseignant doit également soutenir les efforts des participants de se comprendre clairement les uns les autres. Il encouragera chacun à reformuler autrement ce qui est dit pour s'assurer que tous comprennent la même chose et pour éviter que certaines présuppositions demeurent encore implicites.

Il s'agit aussi pour l'enseignant de s'assurer que tous les étudiants puissent porter leur attention sur les questions essentielles, surtout si le groupe est dynamique et familier, car les dérives y sont plus fréquentes. L'enseignant rappelle alors à tous que l'objectif consiste à viser une issue raisonnable, même si les cas discutés prennent souvent la forme d'aporie. A cet égard, il est souhaitable de créer des groupes relativement homogènes qui n'excèdent pas 12 personnes.

Enfin, l'enseignant pose de nouvelles questions à toutes les réponses fournies, pour relancer la discussion, pour explorer plus en profondeur la situation et les difficultés qu'elle engendre et pour élargir l'horizon afin d'éviter l'arbitraire ou d'imposer son imaginaire.

\section{Les avantages d'une approche socratique}

La pertinence d'utiliser une approche socratique dans l'enseignement de l'éthique est manifeste à partir du moment où l'éthique, ou la philosophie, est conçue comme un processus plutôt que comme une théorie. C'est encore plus valable lorsqu'il s'agit d'enseigner l'éthique clinique, puisqu'il est inutile de chercher des solutions déjà toutes préparées à des situations singulières ou de chercher des théories pour adapter ses propres orientations. Au contraire, la réflexion éthique présuppose un processus infini avec toutes les incertitudes et les ambiguïtés que cela implique et elle postule que toute décision est sujette à une possible révision. Par conséquent, il est nécessaire de commencer l'activité de réflexion par des situations concrètes. Ces situations sont généralement pour l'étudiant en lien avec ses interactions avec les patients et ses collègues et sont souvent sources de malaise, d'irritation ou encore provoquent des sentiments d'indignité ou d'injustice. Au lieu de nier l'expérience concrète de l'étudiant, c'est à partir d'elle que l'enseignement se construit.

L'approche socratique reflète la démarche réflexive qui s'engage chez tout individu, tout comme d'ailleurs dans les débats de société. Le médecin, lorsqu'il est confronté, dans sa pratique clinique, à des situations qui éveillent en lui une réflexion éthique, entreprend spontanément un dialogue avec lui-même et avec ses pairs. L'approche socratique reproduit cette mise en œuvre du dialogue qui s'effectue, ici et maintenant, au chevet du patient. Cette approche est l'équivalent logique du dialogue intérieur que l'esprit développe lorsqu'il accroît ses habiletés à la pensée critique, ce qui permet à Minsky ${ }^{7}$ de décrire l'esprit comme une société où chaque pensée entreprend un dialogue avec les autres pensées, de sorte que les contributions des étudiants sont comme les pensées de l'esprit. Toutes les pensées doivent être abordées attentivement et équitablement. De plus, cette approche reflète notre société de plus en plus démocratique et de moins en moins républicaine.

Il ne s'agit pas pour l'enseignant, comme dans un modèle républicain, de transmettre un ensemble de connaissances canoniques qui nous préexisteraient comme chez Platon, mais plutôt de poser les conditions qui encourageront les étudiants à construire ces connaissances dans une communauté de recherche. Comme le souligne Dewey, il s'agit d'intégrer, dans la classe, le débat démocratique sur la vie bonne et juste. En ce sens, la démocratie n'est pas le pouvoir de la masse, mais la puissance du divers. Dans ce contexte, l'enseignant n'est plus un érudit, mais représente le modèle d'un citoyen exemplaire qui s'interroge sur les modes de vie tant personnels que politiques. Son idéal est la réflexivité, habitué qu'il est à la délibération constante. A son contact, les étudiants apprennent à poser des ques- 


\section{Concepts et Innovations}

tions et non à vouloir obtenir à tout prix des réponses. On voit alors que la place de la discussion devient essentielle.

L'avantage le plus important d'une approche socratique est l'accent mis sur l'autonomie de l'individu. L'approche socratique se veut un exercice de subjectivation où l'individu apprend à penser par lui-même à travers la force de décentrement qu'exerce la diversité des points de vue présentés par les autres. En ce sens, penser, c'est chercher le chemin qui nous ferait accéder à soi, car l'autonomie n'est pas donnée, mais acquise par la médiation d'autrui.

Dès lors, l' « éthique de la vertu » devient une condition nécessaire pour le déploiement d'une approche socratique. La vertu nécessaire est principalement « la vertu démocratique, l'habileté à délibérer de manière tolérante et rationnelle, sans répression ni discrimination, en vue de la justice et du bien ». Selon cette approche, l'école, en l'occurrence l'université, devient alors le reflet d'une société démocratique et le lieu de formation à la citoyenneté pour une société plus démocratique. Par conséquent, "l'humanisme de notre temps ne provient plus d'une structure transmise abstraitement, il est construit sur le terrain, chaque jour $»^{1}$.

Même si les interactions dans le milieu médical sont souvent caractérisées par une structure hiérarchique en raison de l'ancien modèle, qui a toujours sa pertinence lorsqu'il s'agit d'acquérir les connaissances scientifiques et cliniques propres à la médecine, lorsqu'il s'agit d'une discussion éthique, il est préférable de favoriser un modèle d'égalité dans la communication, où l'autorité ne relève pas de la domination, mais de la reconnaissance de l'aptitude à bien mener une discussion. L'approche socratique devient une occasion de premier plan pour l'expression de l'empathie et pour l'apprentissage à respecter des points de vue différents, sans toutefois être obligé de faire l'abnégation d'une pensée critique. De plus, le processus dialogique que l'on retrouve dans une approche socratique de l'enseignement favorise le déploiement de l'autonomie des personnes, ce qui ne fait qu'augmenter le niveau de satisfaction des étudiants, de même que leur curiosité et leur réceptivité, sans compter une meilleure tolérance à l'incertitude et au silence.

\section{Conclusion}

Cette stratégie d'apprentissage, avec son accent sur l'interaction de groupe, sur la discussion, sur la résolution de problème, encourage un mouvement de recul vis-à-vis du processus de prise de décision monologique dans lequel les participants cherchent à sanctionner un ensemble de standards absolus qui convergent vers une seule interprétation adéquate et vers la prescription d'un cours d'action déterminé. Cette stratégie vise plutôt à encourager le déploiement d'un processus dialogique qui prend en compte les divergences de pensée et favorise une variété d'interventions possibles.

Puisqu'il s'agit pour l'élève, comme pour l'individu, de réduire l'écart entre ce qui " est " et ce qui " devrait être ", entre les besoins et les désirs et les moyens proposés pour les satisfaire, il devra choisir les options les plus raisonnables, celles qui visent le juste milieu pour réduire justement l'écart. Pour ce faire, ce n'est pas tant de connaissances strictement spéculatives dont l'élève a besoin que de sagesse, quoique cela n'exclue aucunement la prise de contact avec les grandes œuvres. Et la sagesse, comprise comme une harmonisation de l'écart entre les besoins, les désirs et les moyens qui sont proposés pour les satisfaire, est du domaine de l'éthique dans la mesure où elle émerge d'un dialogue avec nous-mêmes et autrui.

\section{Références}

1. Leroux G. Instruire, enseigner, former. Le métier d'enseignant aujourd'hui. Médiane 2006;1:36-49.

2. MacIntyre A. After Virtue, Notre Dame (IN) : University of Notre Dame Press, 1984.

3. Hafferty $F W$, Franks $R$. The hidden curriculum, ethics teaching, and the structure of medical education. Acad Med 1994;64:861-71.

4. Beauchamp TL, Childress JF. Principles of Biomedical Ethics, 5th ed. Oxford: Oxford University Press, 2001.

5. Nelson L. Socratic Method and Critical Philosophy. Selected Essays by Leonard Nelson, New York: Dover, 1965.

6. Heckmann G. Das sokratische Gespräch. Erfahrungen in philosophischen Hochschulseminaren. Hannover: Schroedel, 1981.

7. Minsky M. The Society of Mind, New York : Simon and Schuster, 1986.

Manuscrit reçu le 13 février 2008; commentaires éditoriaux formulés à l'auteur le 2 juin 2008 ; accepté pour publication le 6 juin 2008. 